\title{
All-optical switching with pulsed signals in an all- semiconductor-optical-amplifier loop device
}

\author{
J.-H. Lee ${ }^{\text {a }}$, C.C. Yang ${ }^{\text {a,* }}$, M.C. Shih ${ }^{\text {b }}$, T.J. Chuang ${ }^{\text {c,d }}$ \\ a Department of Electrical Engineering and Graduate Institute of Optical Engineering, National Taiwan University, 1, Roosevelt Road, \\ Sec. 4, Taipei, Taiwan, ROC \\ ${ }^{\mathrm{b}}$ Institute of Optical Sciences, National Taiwan Ocean University, Keelung, Taiwan, ROC \\ ${ }^{\mathrm{c}}$ Center for Condensed Matter Sciences, National Taiwan University, Taipei, Taiwan, ROC \\ ${ }^{\mathrm{d}}$ Institute of Atomic and Molecular Sciences, Academia Sinica, Taipei, Taiwan, ROC
}

Received 20 June 2000; accepted 15 September 2000

\begin{abstract}
Picosecond pulsed-signal operation for efficient self- and cross-switching in an all-semiconductor-optical-amplifier loop device was implemented. Although the configuration of the device is similar to a conventional nonlinear optical loop mirror, the operation principles are quite different. For $\mathrm{cw}$ signals, the device operation relies on the nonlinear coupling effect in a multi-mode interference waveguide amplifier and the lateral field redistribution and amplification by the active loop. However, for pulsed signals the crucial factor of asymmetric gain distribution in the loop needs to be added for the device operation. The pump-probe experiments provided efficient cross-switching results and indicated that GHz-range operation is feasible by redesigning the device configuration for pump signal injection. ( 2000 Elsevier Science B.V. All rights reserved.
\end{abstract}

Nonlinear optical loop mirrors (NOLMs) and their derivatives are important optoelectronics devices for all-optical switching with the applications to high-speed signal processing and optical communications [1-5]. Typically, such a device consists of either an all-fiber loop or a fiber loop asymmetrically inserted with a semiconductor optical amplifier (SOA). The fiber loop is connected with a fiber coupler for input/output coupling. The required optical nonlinearity for all-optical switching comes from either the Kerr effect in fiber

\footnotetext{
${ }^{*}$ Corresponding author. Tel.: +886-2-2365-7624; fax: +8862-2365-2637.

E-mail address: ccy@cc.ee.ntu.edu.tw (C.C. Yang).
}

or gain saturation in the SOA. The fiber loop length of such a device ranges from a few tenths to a few meters, which corresponds to a latency of $>1$ ns. Recently, all-semiconductor devices with the NOLM configuration have received attention for their advantages of low latency, easy integration, compactness, mass production, and low cost [6-8].

In this letter, we report the experimental results of all-optical switching in an all-SOA loop device with pulsed signals. These results represent a significant progress of ours in realizing a practically useful device. The experimental results and theoretical modeling with cw-signal operation of such a device have been reported in Refs. [7,8]. In such a device, a multi-mode interference waveguide 
amplifier (MMIWA) was connected to the SOA loop for input/output coupling. In the cw operation, it was found that the observed nonlinear switching came from the combined effect of the nonlinear coupling in the MMIWA, and the lateral wave field redistribution plus amplification caused by the loop. In this situation, the loop asymmetry did not contribute to the observed nonlinear switching phenomena. However, in the pulsedsignal operation the loop asymmetry becomes crucially important.

Fig. 1 shows the layout of the device. The active loop of $300 \mu \mathrm{m}$ in radius is formed with a curved ridge waveguide with a ridge width of $4 \mu \mathrm{m}$ (a single-mode waveguide). The loop is connected to an MMIWA with a length of $460 \mu \mathrm{m}$ and a ridge width of $8 \mu \mathrm{m}$. The MMIWA serves the function of the coupler with the nonlinear coupling effect. Then, the input and output legs are formed with $4 \mu \mathrm{m}$ wide SOAs. Both have the lengths of about $600 \mu \mathrm{m}$. The device was fabricated on a fourperiod GaAs/AlGaAs multiple quantum well epitaxial structure. The GaAs wells (10 nm thick) and $\mathrm{Al}_{0.25} \mathrm{Ga}_{0.75} \mathrm{As}$ barriers $(20 \mathrm{~nm})$ were sandwiched by two $140 \mathrm{~nm}$ thick $\mathrm{i}-\mathrm{Al}_{0.25} \mathrm{Ga}_{0.75} \mathrm{As}$ separate confinement layers, and $\mathrm{p}-\mathrm{Al}_{0.6} \mathrm{Ga}_{0.4} \mathrm{As}$ and $\mathrm{n}-\mathrm{Al}_{0.6} \mathrm{Ga}_{0.4} \mathrm{As}$ cladding layers. Electro-luminescence measurements indicated that the gain peaks

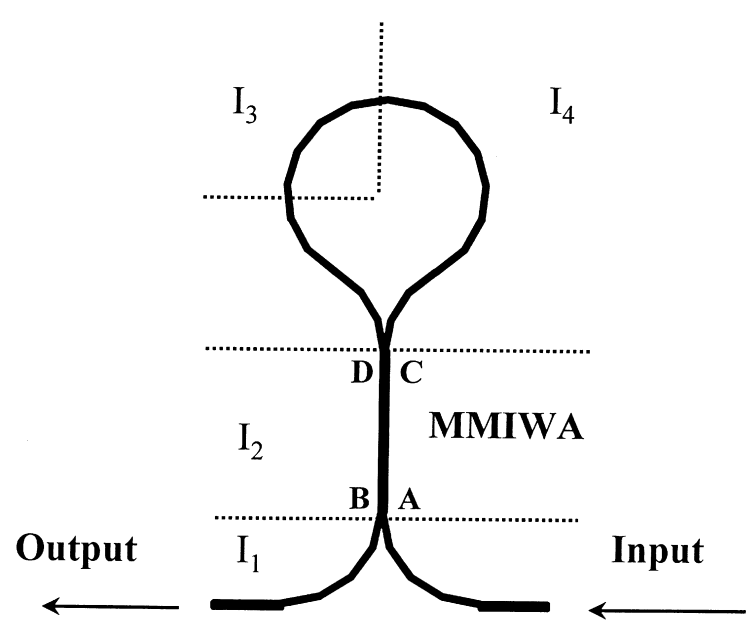

Fig. 1. Schematic diagram of the all-semiconductor-opticalamplifier loop device. The electrode pad is divided into four disconnected sections denoted by $I_{1}$ through $I_{4}$. in the transverse electric (TE) and transverse magnetic (TM) directions were 834 and $817 \mathrm{~nm}$, respectively. The fabrication procedures were the same as the typical processes for semiconductor lasers except the etching technique for forming the ridge waveguides. To achieve vertical and smooth etched walls for reducing bending loss and to minimize surface damage, the UV laser-assisted cryo-etching technique was used [9]. The etching depth was $1.6 \mu \mathrm{m}$. For injecting different currents into different areas, we divided the electro-pad into four disconnected regions, indicated by the injection currents $I_{1}$ (input-output region), $I_{2}$ (MMIWA), $I_{3}$ (one-quarter of the loop), and $I_{4}$ (three-quarter of the loop).

In experiments, 100-fs pulses from a modelocked Ti:sapphire laser were first passed through a monochromator for selecting the required center wavelengths and spectral widths of input signals. With a fixed center wavelength of the fs pulse from the laser, the allowed wavelength range for selection was about $10 \mathrm{~nm}$. After the monochromator, the pulse of a selected wavelength had a width of about 1.4 ps. Such signals were end coupled into the input leg of the device. The signals emergent from the output leg were passed through another monochromator for filtering out amplified spontaneous emission noise. Other details of the experimental procedures can be found in Ref. [8]. Fig. 2 provides three sets of data showing powerdependent switching when the TE-polarized input signals at $834 \mathrm{~nm}$ (TE gain peak) were used. Note that in Figs. 2-5, the continuous curves are drawn to best fit the data points for guiding the eyes. The horizontal axis represents the average input power before entering the input leg. The injection current values of $I_{1}, I_{3}$, and $I_{4}$ were fixed at 40,200, and $100 \mathrm{~mA}$, respectively. Note that this arrangement leads to the injection current density ratio of the one-quarter section over the three-quarter section of the loop to be about $6: 1$. The three curves in Fig. 2 correspond to the $I_{2}$ values (into the MMIWA region) at 25, 50, and $75 \mathrm{~mA}$, respectively. One can see that significant nonlinear switching occurs when $I_{2}$ is sufficiently large. Generally speaking, a larger $I_{2}$ leads to a smaller switching power. It is noted that the input power level in Fig. 2 is two orders of magnitude smaller 


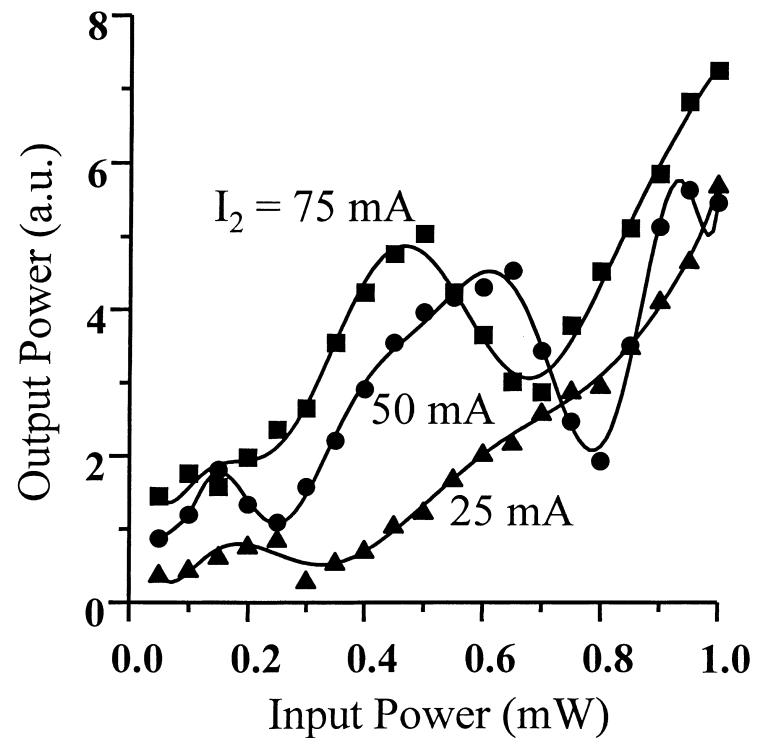

Fig. 2. Output power level as a function of input power with different $I_{2}$ values. The input signal is TE polarized at $834 \mathrm{~nm}$. $I_{1}=40 \mathrm{~mA}, I_{3}=200 \mathrm{~mA}$, and $I_{4}=100 \mathrm{~mA}$.

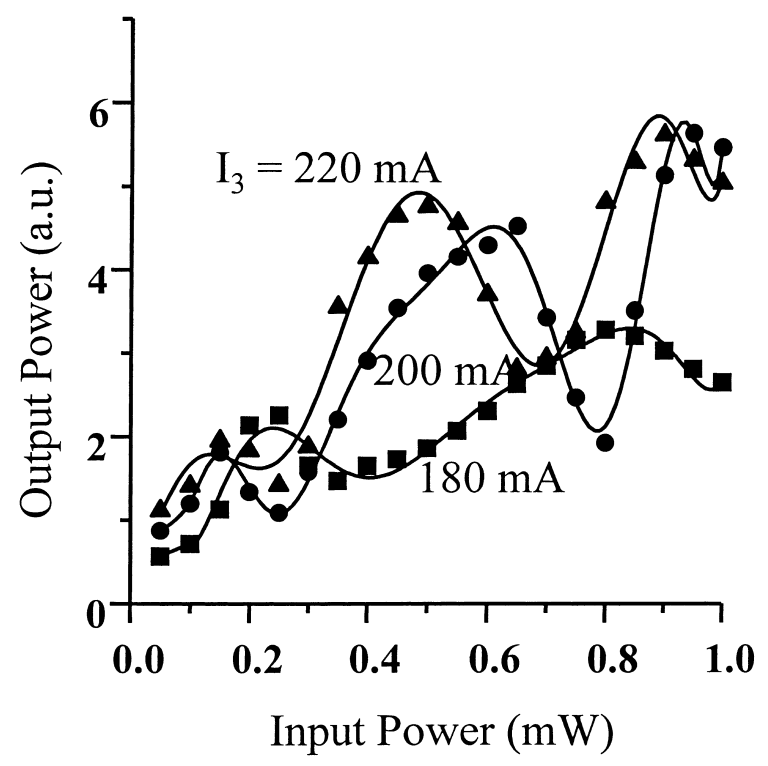

Fig. 3. Output power level as a function of input power with different $I_{3}$ values. The input signal is TE polarized at $834 \mathrm{~nm}$. $I_{1}=40 \mathrm{~mA}, I_{2}=50 \mathrm{~mA}$, and $I_{4}=100 \mathrm{~mA}$.

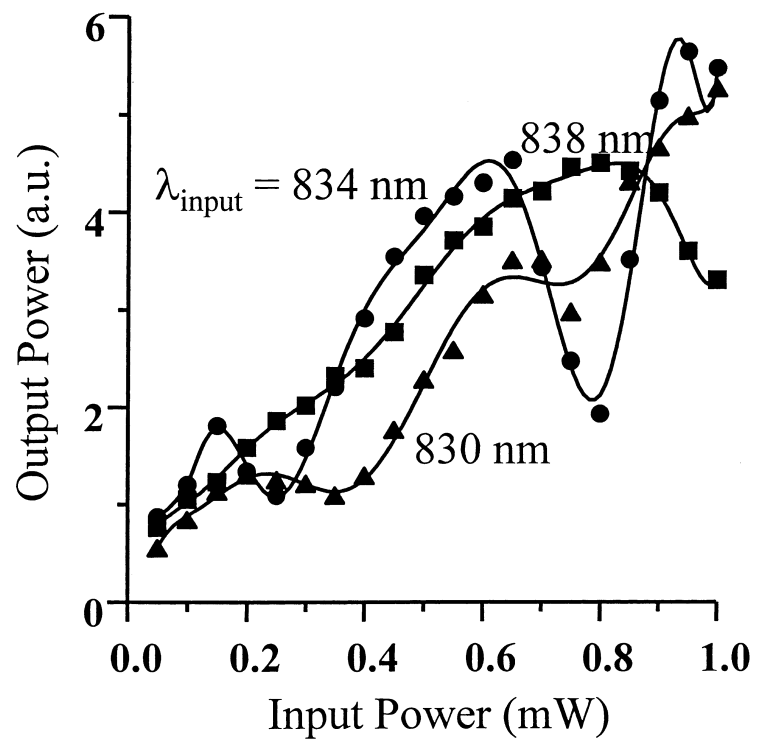

Fig. 4. Output power level as a function of input power with different signal wavelengths. The input signal is TE polarized. $I_{1}=40 \mathrm{~mA}, I_{2}=50 \mathrm{~mA}, I_{3}=200 \mathrm{~mA}$, and $I_{4}=100 \mathrm{~mA}$.

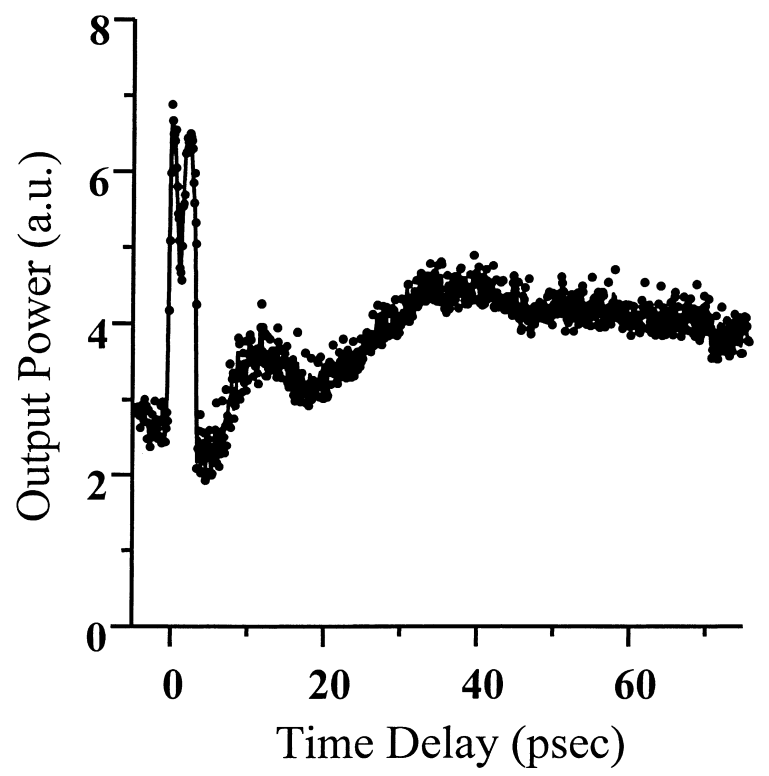

Fig. 5. Probe signal power level as a function of the time delay from a pump-probe experiment. The pump is TM polarized at $825 \mathrm{~nm}$ and has a power of $0.75 \mathrm{~mW}$. The probe is TE polarized at $834 \mathrm{~nm}$ and has a power of $0.25 \mathrm{~mW} . I_{1}=40 \mathrm{~mA}, I_{2}=50$ $\mathrm{mA}, I_{3}=200 \mathrm{~mA}$, and $I_{4}=100 \mathrm{~mA}$. 
than its counterpart in Ref. [8] (see Fig. 4 of this paper), confirming that the pulsed-signal operation is much more efficient for all-optical switching. For instance, $0.6 \mathrm{~mW}$ switching power in the horizontal axis of Fig. 2 corresponds to a switching energy of $79 \mathrm{fJ}$ if $1 \%$ of input coupling efficiency is assumed.

Fig. 3 demonstrates three sets of data with $I_{3}$ varied from 180 to $220 \mathrm{~mA}$. In this figure, $I_{1}, I_{2}$ and $I_{4}$ were fixed at 40,50 and $100 \mathrm{~mA}$, respectively. The signal was still TE polarized with the center wavelength at $834 \mathrm{~nm}$. The increase of $I_{3}$ implies the increase of the loop asymmetry and hence the nonlinear switching efficiency, as verified by the results in Fig. 3. Then, Fig. 4 demonstrates the nonlinear switching results at different signal wavelengths. With $I_{1}, I_{2}, I_{3}$, and $I_{4}$ fixed at 40, 50, 200 , and $100 \mathrm{~mA}$, respectively, the results of three wavelengths of the TE-polarized signal at 830, 834, and $838 \mathrm{~nm}$ are shown. Here, one can see that wavelength choice is quite critical for efficient nonlinear switching. Efficient switching can be observed only near the gain peak.

To observe the cross-switching effect, we prepared two ps pulses for pump-probe experiments. Fig. 5 shows a typical result of the pump-probe experiments for the probe output power level as a function of the pump-probe time delay. Here, the pump was TM polarized at $825 \mathrm{~nm}$ with the input power at $0.75 \mathrm{~mW}$ and the probe was TE polarized at $834 \mathrm{~nm}$ with the input power at $0.25 \mathrm{~mW}$. The injection current values were the same as those in Fig. 4. The zero time delay was approximately set at the first peak of the data curve. One can see the narrow two-hump feature of about $5 \mathrm{ps}$ in width around the zero time delay. It is supposed to correspond to the situation that the pump and probe overlap in most part of the device. After the twohump feature, a long tail in an oscillatory manner can be observed. This long tail is supposed to come from the period of gain recovering from saturation in the MMIWA and loop. Its length should be in the order of carrier lifetime (several hundred ps to a few ns). The oscillatory manner in either the twohump peak or the tail can be attributed to the interferometry nature of the loop device. From several sets of pump-probe data, we can induce that the period of the oscillation increases with time delay. Also, a valley always exists between the peak feature and the long tail. The interpretations for these phenomena require a sophisticated model of the device.

In interpreting the device operation with $\mathrm{cw}$ signals, we considered the nonlinear coupling effect in the MMIWA and the functions of the lateral field redistribution and amplification from the loop [8]. Such considerations are still valid. However, with pulsed signals the loop asymmetry needs to be considered. The nonlinear coupling in the MMIWA results in power-dependent lateral field distribution. After splitting into the two counter-propagating components in the loop, they experience different gain levels and phase shifts in the asymmetric loop. Such differences combine with the nonlinear coupling effect acquired during the backward propagation in the MMIWA to give various distributions of the signal power between the input and output legs. When the pump-probe experiment is conducted, the nonlinear coupling process of the strong pump signal dominates the lateral field distribution of the probe signal in the MMIWA. Also, the asymmetric gain saturation due to the counter-propagating pump signals may enhance the asymmetric gain and phase factors for the counter-propagating probe signals. Such complicated processes result in the observed cross-switching phenomenon, shown in Fig. 5.

In summary, we have demonstrated the pulsedsignal operation in an all-SOA loop device. Efficient all-optical switching was implemented. The pulsed-signal operation of the device was quite different from that of $\mathrm{cw}$ signals. Besides the lateral field redistribution and amplification by the loop, and the nonlinear coupling effect in the MMIWA, the asymmetry in gain distribution along the loop was crucially important for the pulsed operation. Experimental results have shown quite efficient self- and cross-switching. Further investigations include the modeling of the pulsed operation of such a device and the design of device configuration for reducing the decay time of nonlinear switching [4]. Meanwhile, the fabrication of such a device operated near $1550 \mathrm{~nm}$ would make their applications for fiber communications practical. 


\section{Acknowledgements}

This research was supported by National Science Council, The Republic of China, under the grants of NSC 88-2112-M-002-004, NSC 88-2215E-002-012, and NSC 88-2215-E-002-014.

\section{References}

[1] J. Cai, K. Feng, X.P. Chen, A.E. Willner, Equalization of nonuniform EDFA gain using a fiber-loop mirror, IEEE Photon. Technol. Lett. 9 (1997) 916-918.

[2] W.S. Wong, S. Namiki, M. Margalit, H.A. Haus, P. Ippen, Self-switching of optical pulses in dispersion-imbalanced nonlinear loop mirrors, Opt. Lett. 22 (1997) 11501152.

[3] D. Wang, E.A. Golovchenko, A.N. Pilipetskii, C.R. Menyuk, M.F. Arend, Nonlinear optical loop mirror based on standard communication fiber, J. Lightwave Technol. 15 (1997) 642-646.
[4] J.P. Sokoloff, P.R. Prucnal, I. Glesk, M. Kane, A terahertz optical asymmetric demultiplexer (TOAD), IEEE Photon. Technol. Lett. 5 (1993) 787-790.

[5] M. Eiselt, W. Pieper, H.G. Weber, SLALOM: semiconductor laser amplifier in a loop mirror, J. Lightwave Technol. 13 (1995) 2099-2112.

[6] E. Jahn, N. Agrawal, W. Pieper, H.J. Ehrke, D. Franke, W. Furst, C.M. Weinert, Monolithically integrated nonlinear Sagnac interferometer and its application as a 20 Gbit/s all-optical demultiplexer, Electron. Lett. 32 (1996) 782-784.

[7] J.H. Lee, D.A. Wang, H.J. Chiang, D.W. Huang, S. Gurtler, C.C. Yang, Y.W. Kiang, B.B. Chen, M.C. Shih, T.J. Chuang, Nonlinear switching in an all-semiconductoroptical-amplifier loop device, IEEE Photon. Technol. Lett. 11 (1999) 236-238.

[8] J.H. Lee, D.A. Wang, Y.W. Kiang, H.J. Chiang, C.C. Yang, Nonlinear switching behaviors in a compact allsemiconductor-optical-amplifier Sagnac interferometer device, IEEE J. Quant. Electron. 35 (1999) 1469-1477.

[9] C.-H. Tsai, J.-H. Lee, H.-J. Chiang, C.C. Yang, M.C. Shih, B.C. Chen, T.J. Chuang, Y. Chang, Semiconductor circular ring lasers fabricated with the cryo-etching technique, IEEE Photon. Technol. Lett. 10 (1998) 751-753. 